\title{
CLOUD-BASED ENERGY MONITORING AND SIMULATION IOT PLATFORM
}

\author{
George SUCIU \\ Beia Consult International \\ george@beia.ro \\ Lucian NECULA \\ Beia Consult International \\ lucian.necula@beia.ro \\ Ioana ROGOJANU \\ Beia Consult International \\ ioana.rogojanu@beia.ro
}

\begin{abstract}
Nowadays, most organizations that aim to optimize their energy consumption are experiencing a lack of Business Intelligence (BI) solutions for supporting their decision-making process in order to implement viable enterprise systems for energy-efficiency. BI technologies can include data storage, querying, data processing, rule-based simulation, and advanced visualization capabilities, including processing data from the Internet of Things (IoT) monitoring platforms. Thus, most of companies face different challenges in monitoring the energy consumption inside buildings since each company has to face an unique combination of factors which needs to be properly evaluated. Another degree of complexity is added if the company uses energy production solutions since the reliability of the investment is influenced by the energy demand of the company, changes in the consumption patterns, local weather conditions and unforeseen costs related to the exploitation of the equipment. This paper provides an example of a Cloud-based BI energy monitoring and simulation platform which aims to help organizations monitor and forecast the energy production and consumption within a building and to simulate the economic efficiency for multiple investment scenarios. Also, it presents an overview on the Verbund device, which was used to measure the power consumption inside the building, and MQTT (Message Queuing Telemetry Transport) protocol which was used to collect the data from the device and to display data in the Grafana dashboard.
\end{abstract}

Keywords: Business Intelligence tool, Cloud-based platform, Smart energy, IoT

JEL classification: C87, K32, L84

DOI: $10.12948 / \mathrm{ie} 2019.06 .07$

\section{Introduction}

Nowadays, in order to address the optimization of energy consumption and exploitation cost reduction, companies concern themselves with the integration of innovative energy efficiency solutions for the foreseeable future demand for energy. Considering the challenge of optimizing energy consumption, the use of information and communications technology (ICT) plays an essential role in the management decision for both energy providers and consumers [1]. As a result of the market demand in the energy efficiency domain, commercial Energy Service Companies (ESCOs) provide a wide range of BI tools for the energy sector, including solutions for power generation and energy supplying, energy conservation, risk calculation, energy efficiency assessment and management etc [2]. ESCOs are offering services similar to Energy Service Providing Companies (ESPCs), but, in contrast to them, the difference lays on the 
concept of performance-based contracting. In the case of ESCOs, the payment for the products and services is directly linked to the amount of saved energy [3]. Energy Performance Contracts (EPC) commonly start with an audit for the examination of the sources of inefficiency, so ESCOs can formulate the strategy for energy efficiency based on all internal and external factors that may threaten the feasibility of the project [4], [5]. In this context, the Internet of Things technologies facilitate the design and development of innovative solutions which can provide ESCOs with more control over the utility operations. Simulation tools and business analytics can be used together to determine the impact of various factors, such as energy prices, local weather conditions, energy production and consumption in terms of middle and long-term economic effects of investment decisions [6]. BI provides a set of techniques and processes used for transforming the raw data into useful information with a high impact in the field of Business Analysis. BI tools are designed to identify new opportunities and to substantiate strategies based on the existing data. Thus, the main advantage of using BI tools is that it allows to simulate, analyze and manipulate huge volumes of data into useful information for the decision-making process [7]. The CitiSim platform [8] consists of a Smart City monitoring and simulation solution which provides high-level tools for various domains, such as energy optimization, emergencies management and mobility management. In the context of CitiSim project, this article presents an innovative Internet of Things (IoT) and Cloud-based platform which uses data collected by sensors and energy production forecasting data to simulate economic efficiency of investment decisions using user-defined scenarios.

The rest of the paper is organized as follows: Section II presents several BI tools with applicability in analyzing the energy efficiency, Section III depicts the most common device used to collect data for simulation, Section IV presents CitiSim IoT Energy Monitoring and Simulation platform developed within CitiSim project, while Section IV concludes the paper.

\section{Related work}

Companies keep searching for new approaches in terms of energy consumption. In this case, a wide range of programs has been developed for different purposes, such as data visualization, data monitoring, business analytics for optimization of energy costs.

In this section, we investigate related work regarding BI tools for analyzing energy efficiency based on data from IoT solutions for monitoring energy consumption.

\subsection{QlikView}

QlikView [9] is one of the most flexible BI tools for manipulating data. The analytic solution allows users to build and develop interactive dashboards and analytic applications rapidly, using guided analytics. The data integration layer is loaded from multiple sources and transformed into useful information. Therefore, the need for external data repositories or tools is reduced [10].

\subsection{Spotfire}

TIBCO Spotfire is a software designed for data analytics and data exploration. It enables the user to analyze the data using predictive statistics [11]. Spotfire is used in a practical and userfriendly platform for developing an interactive representation of charts. The main advantage of using Spotfire is that it allows real-time data visualization [12].

\subsection{Tableau}

Tableau [13] is a BI and data visualization tool which allows the users to create interactive reports which can help the organizations in the process of connecting and sharing their data. The tool enables the user to integrate different Application Programming Interfaces (APIs) for increasing the performances of the analysis. 
www.conferenceie.ase.ro

\subsection{IBM Cognos}

IBM Cognos is a Cloud-based BI tool which includes many features like data monitoring, analysis of information and reporting the results in a unified environment [14]. The data can be explored using embedded geospatial-mapping capabilities in the dashboarding function [15].

\subsection{SAP Business Intelligence}

SAP Business Intelligence [16] is a Cloud-based BI tool which focuses on providing collaborative planning using predictive analytics and Machine Learning (ML) techniques. The integrated ML tools simplify access to information using Natural Language Processing (NLP) technologies for modeling complex scenarios.

\section{Energy Monitoring}

Optimizing the electrical power consumption is one of the fundamental goals of smart energy management. Measuring the electrical power is the first step to get an idea regarding the consumption inside a building and, consequently, to adopt strategies for energy savings and efficiency [17]. Traditional energy meters for billing the energy consumption have been replaced by Smart Meters which, besides billing, enable users to monitor their energy consumption [18]. This section describes the device used for measuring the power consumption inside a building. Verbund energy sensor (figure 1) allows the user to monitor both energy production and consumption.

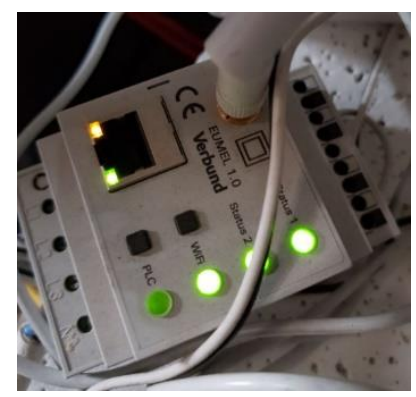

Figure 1. Verbund energy sensor implemented for monitoring energy production and consumption within BEIA's headquarters

The Verbund device records the total power consumption and transmits the data further by accessing JSON link address REST/JSON. However, this request can only be sent in the same WiFi network. The information from Verbund can be transferred via MQTT protocol to a server (mqtt.beia-telemetrie.ro). Using the Grafana platform, the necessary parameters are displayed as a graph for a better understanding of the events which take place at the inverter level. In order to communicate with the Power-Meter, the users' application needs to have an HTTP request standard including basic authentication to one of the Power-Meter communication interfaces such as WiFi, wired Ethernet or powerline communication [19]. Figure 2 presents the power consumption from Verbund device in Grafana dashboard.

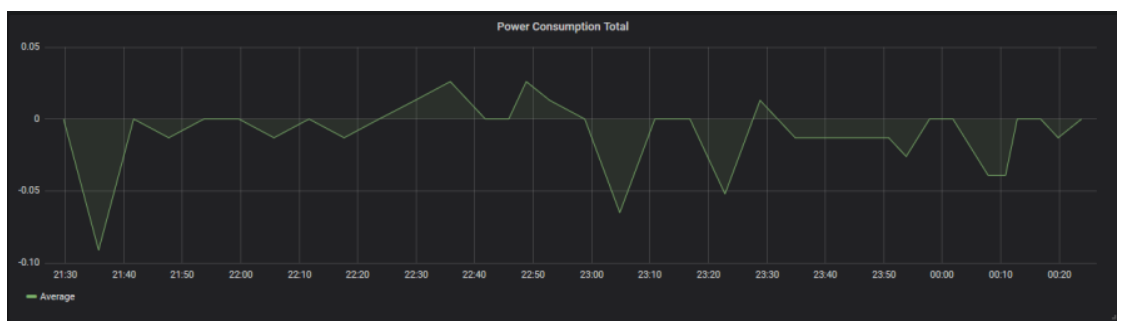

Figure 2. Total power consumption Verbund energy sensor in Grafana 
www.conferenceie.ase.ro

\section{Implementation of Business Intelligence tool}

The primary purpose of the platform is to evaluate the economic performance perspectives in terms of energy efficiency for various user-defined scenarios.

The CitiSim Smart Energy Business Intelligence tool, presented in figure 3, relies on three functionalities:

- monitoring the production and consumption of energy, based on IoT devices, MQTT protocol [20] and Grafana platform for data visualization [21];

- energy production simulation based on EnergyPlus and weather data collected from different devices;

- economic performance simulation of energy efficiency for various investment scenarios.

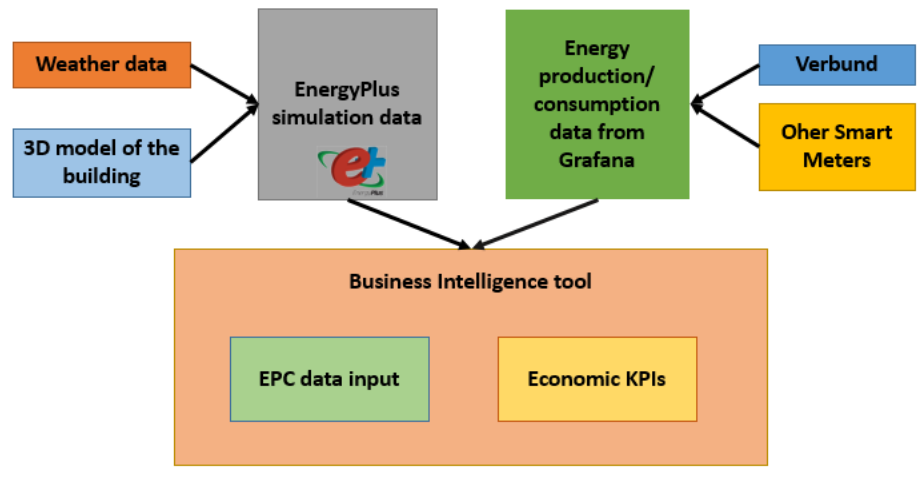

Figure 3. CitiSim Smart Energy Business Intelligence tools

The BI tool relies on the data collected from Smart Meters (from Verbund in this case, but other smart meters can be used) and the user input regarding the predefined scenarios. The data for the simulation is provided by the user, based on the past energy bills or data collected by sensors. The architecture of CitiSim BI tool is presented in figure 4.

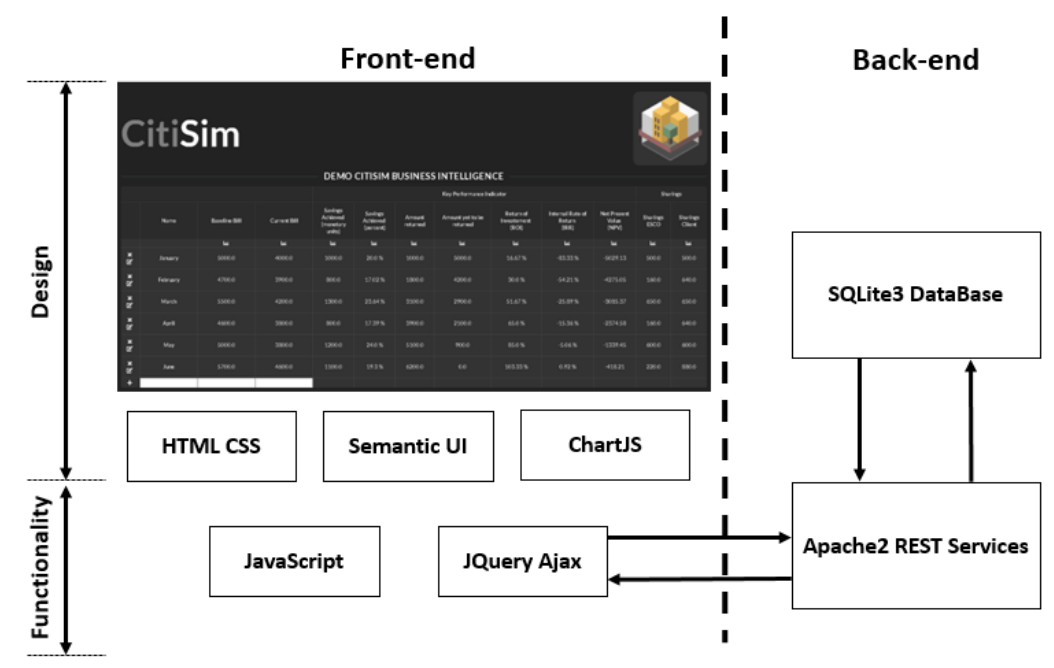

Figure 4. BI tool architecture

For simulation based on a user-defined scenario, the CitiSim BI tool was developed using web a Representational State Transfer (REST) architecture.

Front-end components:

- SemantincUI was used for the structure and the styling of each page;

- CharJS for the graphical representation; 
www.conferenceie.ase.ro

- Query and Ajax for asynchronous calls of the back-end services.

Back-end components:

- SQLite3 database;

- Python Flask Rest services for data processing.

The BI tool provides scenario-based key performance indicators (KPIs) such as Net Present Value (NPV), Internal Rate of Return (IRR), Return of Investment (ROI) and the distribution of savings between the ESCO and the client. The KPIs can be visualized using graphs and charts, thus the values from multiple scenarios can be compared.

\section{Conclusions}

The Cloud-based smart energy platform developed within the CitiSim project aims to provide means that will increase energy efficiency of the buildings. The integration between the platform's components (energy monitoring, energy simulation, and business analytics) determines the system to operate as a toolchain. In this context, CitiSim platform provides a viable solution for generating awareness regarding energy consumption, energy production potential and economic KPIs.

As future work, the platform will be upgraded with new features such as support for other smart meters existing on the market and a web page for activation of an actuating unit such as a smart circuit breaker.

\section{Acknowledgment}

This paper was partially supported by UEFISCDI Romania and MCI through projects CitiSim, Power2SME, 3DSafeGuard, PARFAIT and funded in part by European Union's Horizon 2020 research and innovation program under grant agreements No. 777996 (SealedGRID project) and No. 787002 (SAFECARE project).

\section{References}

[1] A. R. Al-Ali, I. A. Zualkernan, M. Rashid, R. Gupta, and M. Alikarar, "A smart home energy management system using IoT and big data analytics approach". IEEE Transactions on Consumer Electronics, 63(4), 426-434, 2017.

[2] K. Alayón and G. Johansson, "Conceptual sustainable production principles in practice: do they reflect what companies do?", in Journal of Cleaner Production, 141, pp. 693-701, 2017.

[3] W. TariqA, M. L. OthmanB, N. I. A. WahabC, and M. EbrahimD “A Review on ESCO's Challenges and Project Management as a solving tool" Indonesian Journal of Electrical Engineering and Computer Science, 12(1), 269-274, 2018.

[4] European Association of Energy Service Companies. The EPC process. Internet: http://euesco.org/about-epc/epc-process/index.html, [Jan. 20, 2019].

[5] G. Suciu, L. Necula, V. Suciu and Y. Curtmola, "Cloud-Based platform for enhancing energy consumption awareness and substantiating the adoption of energy efficiency measures within SMEs" in 2018 14th International Wireless Communications \& Mobile Computing Conference (IWCMC), 2018, pp. 1018-1023.

[6] N. Le Truong, A. Dodoo, L. Gustavsson, "Effects of energy efficiency measures in districtheated buildings on energy supply”, Energy, 142, pp.1114-1127, 2018.

[7] A. Shukla and S. Dhir, "Tools for Data Visualization in Business Intelligence: Case Study Using the Tool Qlikview", Information Systems Design and Intelligent Applications, pp. 319-326, 2016.

[8] Beia Consult International. CitiSim project. Internet: http://www.beiaro.eu/citisim/, [Jan. 20, 2019]. 
[9] QlikView. Internet: https://www.qlik.com/us/products/qlikview, [Jan. 20, 2019].

[10] C. Anderson, "Business Intelligence”, In Data Science in Practice Springer, pp. 97-118, 2019.

[11] Business Intelligence. Internet: businessintelligence.com/bi-vendors/tibco-spotfire, [Jan. 20, 2019].

[12] A. Shukla and S. Dhir "Tools for Data Visualization in Business Intelligence: Case Study Using the Tool Qlikview", Information Systems Design and Intelligent Applications, pp. 319-326, 2016.

[13] Tableau. Internet: https://www.tableau.com/, [Jan. 20, 2019].

[14] White Paper: The IBM Cognos platform: Meeting the needs of both business users and IT, IBM Software Group. Internet: http://www.5xtechnology.com/Portals/92116/docs/ibm_cognos_ platform.pdf, [Jan. 20, 2019].

[15] Cognos Software. Internet: https://www.ibm.com/products/cognos-analytics, [Jan. 20, 2019].

[16] SAP Business Intelligence tool. Internet: https://www.sap.com/products/cloudanalytics.html, [Jan. 20, 2019].

[17] Ipsos MORI. Smart Meter Customer Experience Study: Post-Installation Survey Report; Department for Business, Energy \& Industrial Strategy: London, UK, August 2017. Internet: https://assets.publishing.service.gov.uk/government/uploads/system/uploads/attachment_ data/file/650970/Postinstall_key_findings_report_FINAL_05102017_PUBLICATION.pd f, August, 2017, [Jan. 20, 2019].

[18] S. Singh, A. Yassine, "Big Data Mining of Energy Time Series for Behavioral Analytics and Energy Consumption Forecasting", Energies, 11, pp. 452, 2018.

[19] Verbund. Internet: https://www.verbund.com/-/media/verbund/ueber-verbund/investor relations/finanzpublikationen/en/2018/verbund-dma-management-approach-en.ashx, [Jan. 20, 2019].

[20] Eclipse Mosquitto. MQTT. Internet: http://mosquitto.org/man/mqtt-7.html, [Jan. 20, 2019].

[21] Grafana Labs. Internet: https://grafana.com/grafana, [Jan. 20, 2019]. 